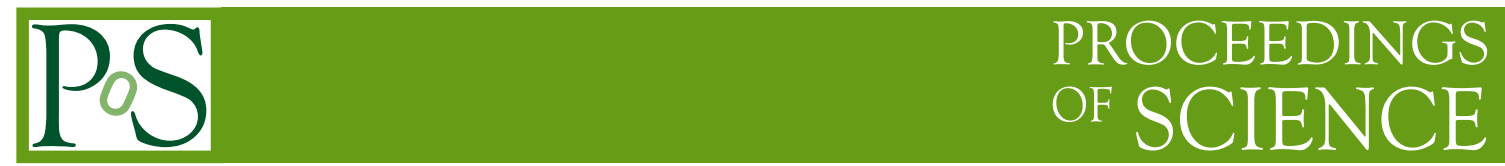

\title{
Search for low-mass new physics at BESIII
}

\author{
Fengyun $\mathbf{L i}^{* \dagger}$ \\ Peking University \\ E-mail: lify@pku.edu.cn
}

With the high luminosity of charmonium states data accumulated by BESIII, a lot of new physics topics have been searched for different motivations or hypothesis that may be possible to explain the physical anomalous observations or test the SM. A summary on recent exotic particles search and SM rare or forbidden decay new physics studies from BESIII are presented in this paper.

VIII International Workshop On Charm Physics

5-9 September, 2016

Bologna, Italy

* Speaker.

${ }^{\dagger}$ On behalf of BESIII collaboration 


\section{Introduction}

Experiencing several years experimental test, SM can explain most of the physical observation and give predictions which then be verified by experiments later. Although the theory and experiment agree well most of time, many experimental results, which can not be included into traditional SM, still remain to be puzzles to us. New physics ideas or theories, even some of which are against with traditional SM , are put forward as the extensions of SM. BESIII is a symmetric $e^{+} e^{-}$collider placed at Beijing Electron Positron Collider (BEPCII) in Beijing, designed for $\tau$-charm physics study at center-of-mass system (CMS) energy from 2 to $5 \mathrm{GeV} / c^{2}$. Since the data taking from 2009, BESIII has collected the world largest data sample around charmonium mass region at CMS of $J / \psi$ (1.3 billion events), $\psi(3686)$ (0.5 million events), $\psi(3770)\left(2.9 \mathrm{fb}^{-1}\right)$ and unique XYZ data. Since most of the new physics effects are highly suppressed and it can only be observed with a greatly high precision experimentally, the high statistics data sample and good detector performance of BESIII make it very promising to search for the signals of new physics beyond SM here. The major topics we focus in this paper at BESIII are the new exotic particles and some SM rare or forbidden decays search.

\section{Exotic particles searches}

The particle family of SM is very large and complicated. Three generations quarks and leptons build the world as elemental blocks. As the conventional quark model description, they made up mesons, baryons and everything of the world. The gauge bosons affect the strong, weak and electromagnetic interactions between particles. Like the fundamental concept of SM, a few new particles are proposed to account for the remained puzzles, like axion, proposed to solve the strong $\mathrm{CP}$ asymmetry, dark photon, proposed for dark matter interactions and mixing with SM matter, light higgs boson, proposed as a member of higgs family, which is introduced in next-to-minimal supersymmetric Standard Model to solve or alleviate the so-called "little hierarchy problem".

The light higgs boson $A^{0}$ is considered to be a product of $J / \psi$ radiative decay and it can decay to a muon pair $\left(J / \psi \rightarrow \gamma A^{0}, A^{0} \rightarrow \mu^{+} \mu^{-}\right)$. BESIII has searched the light higgs boson $A^{0}$ firstly through 106 million $\psi(3686)$ events. [2] Due to the intermediate production of $J / \psi$, non-resonant background can be reduced very effectively. A data sample of $\psi(3686) \rightarrow \pi^{+} \pi^{-} J / \psi, J / \psi \rightarrow$ $\gamma \mu^{+} \mu^{-}$is selected while the major background is from $J / \psi \rightarrow \gamma \pi^{+} \pi^{-}$due to $\mu / \pi$ misidentification, which is suppressed with a strict selection criteria on muon counter (MUC) penetration depth. $M\left(\mu^{+} \mu^{-}\right)$spectrum of selected sample are plotted in Figure 1(a) and no significant $A^{0}$ signal is observed. So the upper limit of number of $A^{0}$ signal is set at the $90 \%$ confidence level in Figure 1 (b) and corresponded branching fraction of $\mathscr{B}\left(J / \psi \rightarrow \gamma A^{0}\right) \times \mathscr{B}\left(A^{0} \rightarrow \mu^{+} \mu^{-}\right)$is plotted in Figure 1(c). The final branching fraction result ranges from $4 \times 10^{-7}$ to $2.1 \times 10^{-5}$.

BESIII also searched the signal of $A^{0}$ directly with 225 million $J / \psi$ data through $J / \psi \rightarrow$ $\gamma \mu^{+} \mu^{-}$. [3] A data sample of $J / \psi \rightarrow \gamma \mu^{+} \mu^{-}$are selected in which the major contamination is from $J / \psi \rightarrow \rho \pi, \gamma f_{2}(1270), \gamma f_{0}(1710)$ for the misidentification between muon and pion. Due to the direct usage of high statistics $J / \psi$ data, this analysis gives a factor of five times improvement result for the upper limit of product branching fraction of $\mathscr{B}\left(J / \psi \rightarrow \gamma A^{0}\right) \times \mathscr{B}\left(A^{0} \rightarrow \mu^{+} \mu^{-}\right)$ ranging between $2.8 \times 10^{-8}$ and $4.953 \times 10^{-6}$ than the previous result with $\psi(3686)$ data. The work 


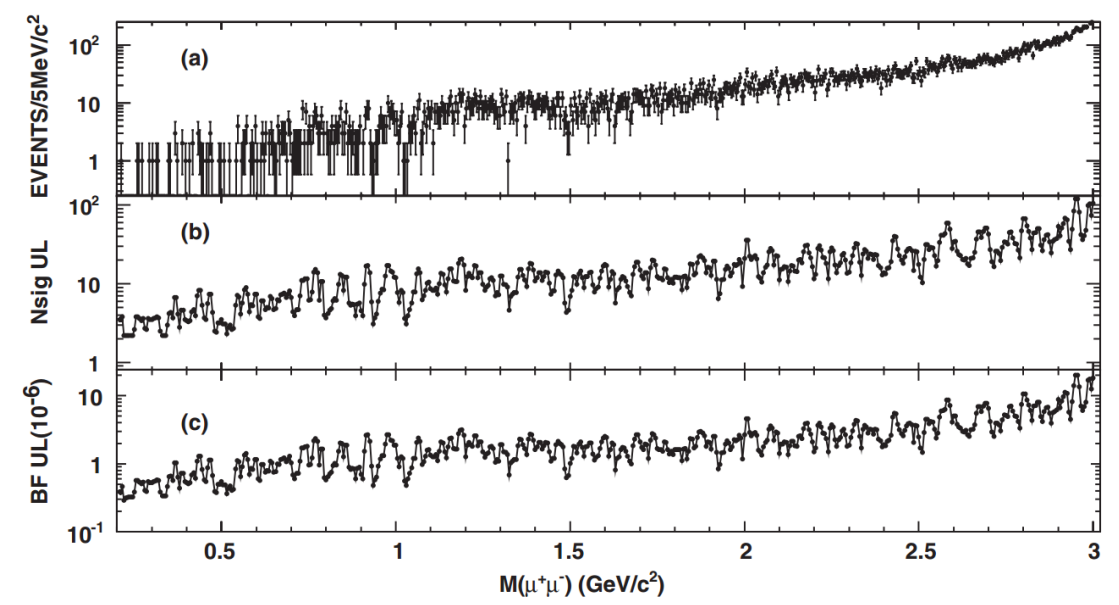

Figure 1: (a) $M\left(\mu^{+} \mu^{-}\right)$spectrum (b) Upper limit of signal event (c) Upper limit of branching fraction $\mathscr{B}\left(J / \psi \rightarrow \gamma A^{0}\right) \times \mathscr{B}\left(A^{0} \rightarrow \mu^{+} \mu^{-}\right)$

also computes the coupling parameter $g_{b}$ and compares BESIII result with BABAR measurement as shown in Figure 2. The new result is complementary and seems to be better than BABAR in the low-mass region for $\tan \beta \leq 0.6$. Combine with BABAR previous result, the measured $\cos \theta_{A} \times \mathscr{B}\left(A^{0} \rightarrow \mu^{+} \mu^{-}\right)$result constrains the $A^{0}$ is mostly a singlet.
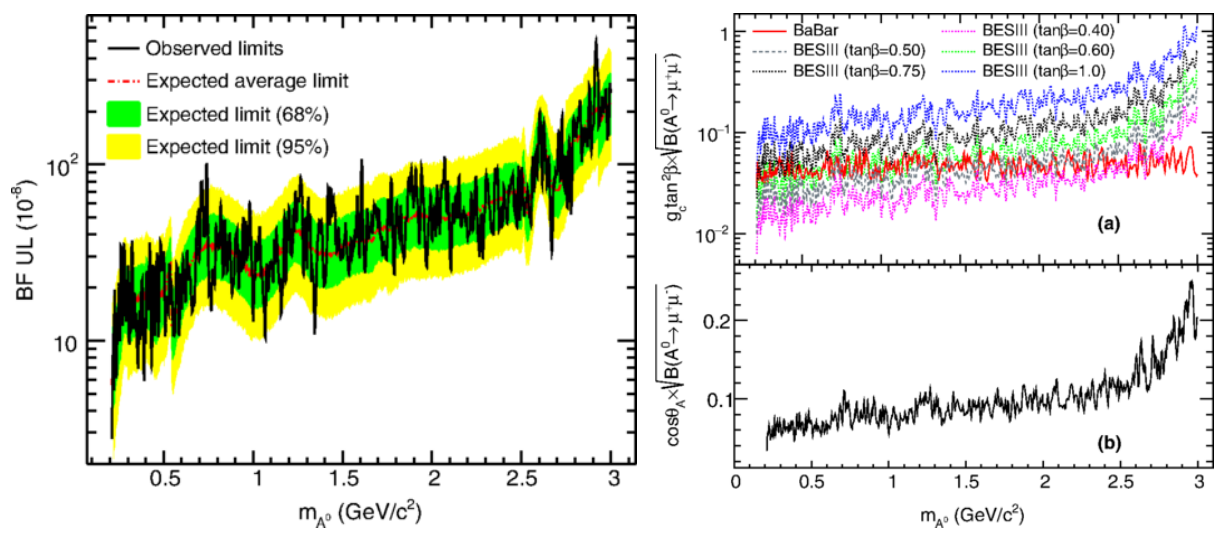

Figure 2: (Left) Upper limit of branching fraction $\mathscr{B}\left(J / \psi \rightarrow \gamma A^{0}\right) \times \mathscr{B}\left(A^{0} \rightarrow \mu^{+} \mu^{-}\right)$(Right) [top] The 90\% C.L. upper limit on $g_{b}\left(=g_{c} \tan ^{2} \beta\right) \times \sqrt{\mathscr{B}\left(A^{0} \rightarrow \mu^{+} \mu^{-}\right)}\left[\right.$bottom] $\cos \theta_{A}\left(=\left|\sqrt{g_{b} g_{c}}\right| \times \sqrt{\mathscr{B}\left(A^{0} \rightarrow \mu^{+} \mu^{-}\right)}\right)$

To explore how dark matter interact with SM matter and with each other themselves, an extra $\mathrm{U}_{1 d}$ gauge symmetry is introduced to expand SM gauge symmetry. The new force brings in another gauge boson denoted as dark photon (Also named as $\mathrm{U}$ boson, $A^{\prime}, \gamma^{\prime} \ldots$ ), which is constrained to be mostly as light as $\mathrm{MeV} / \mathrm{c}^{2}$ to $\mathrm{GeV} / \mathrm{c}^{2}$ mass scale and the main decay mode is through lepton pairs. It can connect to SM sector through a kinematic mixing with SM photon in which the mixing strength is described with parameter $\varepsilon$. With $2.92 \mathrm{fb}^{-1}$ data collected at center-of-mass system (CMS) energy of $\psi(3770)$, BESIII has searched the track of dark photon $\gamma^{\prime}$ with ISR method through both electron and muon final states $e^{+} e^{-} \rightarrow \gamma^{\prime} \gamma_{I S R} \rightarrow l^{+} l^{-} \gamma_{I S R}(l=\mu, e)$. [4] The dark photon is searched in the mass range between 1.5 and $3.4 \mathrm{GeV} / c^{2}$ and no significant signal is observed. Corresponded 
upper limit on the mixing parameter $\varepsilon$ is between $10^{-3}$ and $10^{-4}$ as a function of dark photon mass with a confidence level of $90 \%$ as shown in Figure 3. The limit is competitive in this range with BABAR while only using a much smaller data sample.

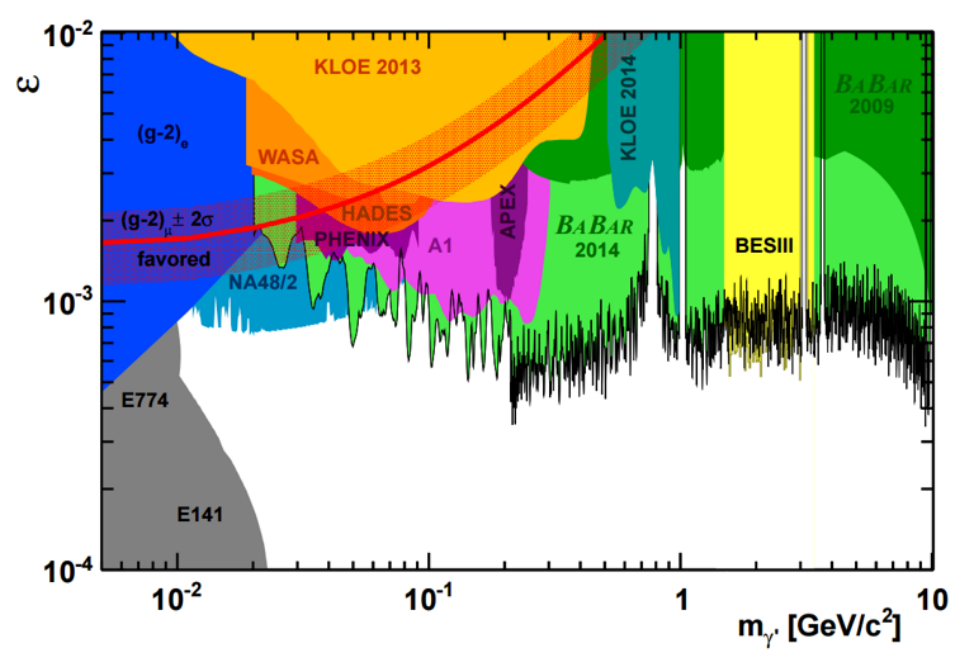

Figure 3: Exclusion limit on the mixing strength $\varepsilon$ and dark photon mass $\mathbf{M}_{\gamma^{\prime}}$

\section{SM rare or forbidden decays}

The law of symmetry and conservation constrains the particle interaction modes. However, some forbidden processes can still happen due to mechanisms like high order contribution, loop intermediation or some new physics particle propagator. Usually just from SM calculation, these process are so highly suppressed that most of them are far beyond present detectors' reach. If we find some enhancement in these rare or forbidden process, the result must indicates some new physics mechanism beyond SM.

Flavour changing neutral current process refers to that a fermion current favour changes without its electric charge. It can only happen beyond tree level and are highly suppressed by the Glashow-Iliopoulos-Maiani (GIM) mechanism. In charm physics, one of the interesting FCNC process is $D^{0} \rightarrow \gamma \gamma$. Theory prediction for branching fraction is $\left(3.5_{-2.6}^{+4.0}\right) \times 10^{-8}$ where long-distance contributions dominate. But some extensions to the SM can enhance FCNC processes by many orders of magnitude. For instance, gluino exchange can increase the branching fraction for the $c \rightarrow u \gamma$ transition to $6 \times 10^{-6}$ in minimal supersymmetric SM. With $2.92 \mathrm{fb}^{-1} \psi(3770)$ data, BESIII uses a double tag method to search for the FCNC process $D^{0} \rightarrow \gamma \gamma$. The tagged $\bar{D}^{0}$ is reconstructed with five hadronic modes $\bar{D}^{0} \rightarrow K^{+} \pi^{-}, K^{+} \pi^{-} \pi^{0}, K^{+} \pi^{-} \pi^{+} \pi^{-}, K^{+} \pi^{-} \pi^{+} \pi^{-} \pi^{0}, K^{+} \pi^{-} \pi^{0} \pi^{0} . M_{B C}$ and $|\Delta E|$ are used to select the tagged $\bar{D}^{0}$ candidate. Then the remained photons are used to reconstruct $D^{0}$. The $\Delta E$ distribution of signal $D^{0}$ and tagged $\bar{D}^{0}$ candidate are plotted in Figure 4 . In the analysis main background $D^{0} \rightarrow \pi^{0} \pi^{0}$ is also measured with a similar tag method and the result is $(8.24 \pm 0.21$ (stat. $) \pm 0.30($ syst. $)) \times 10^{-4}$. The final $90 \%$ confidence level upper limit for FCNC decay branching fraction is $\mathscr{B}\left(D^{0} \rightarrow \gamma \gamma\right)<3.8 \times 10^{-6}$. With only $2.92 \mathrm{fb}^{-1}$ data at open-charm threshold, The result is close to old BABAR result $2.2 \times 10^{-6}$ with $470.5 \mathrm{fb}^{-1}$ [6], which indicates 
BESIII is very competitive in charm decay study. The latest result comes from Belle collaboration, $8.5 \times 10^{-7}$ with $832832 f b^{-1}$ data [7].
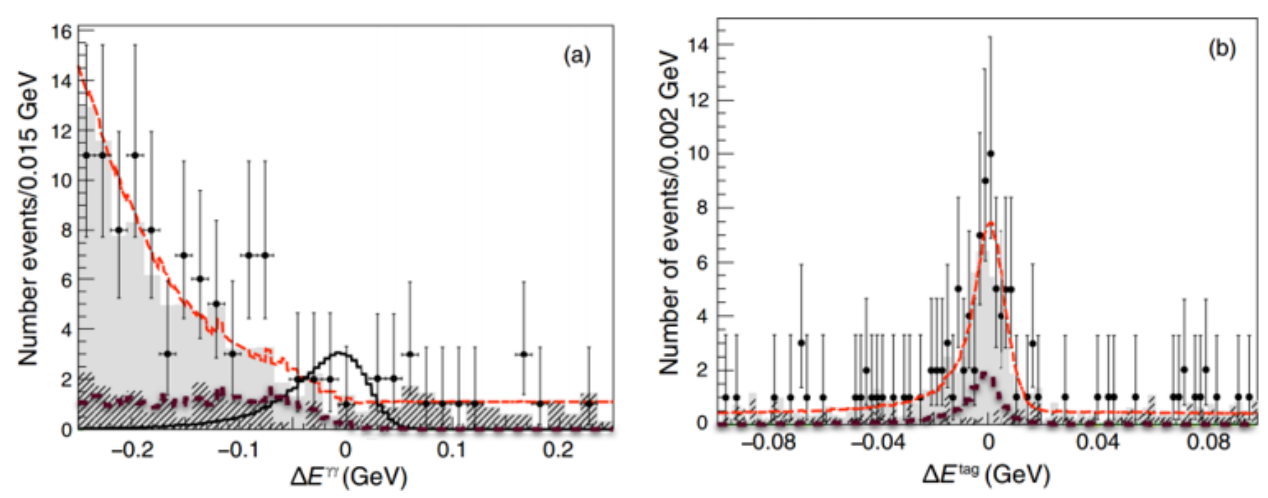

Figure 4: $\Delta E$ distribution for (a) Signal $D^{0}$ candidate (b) Tagged $\bar{D}^{0}$ candidate

Invisible decays of mesons like $J / \psi, \Upsilon$ and other quarkonium states would offer us a window into what may lie beyond the SM of particle physics notably for light dark matter, since apart from neutrinos, the minimal SM predicts no other channels that these states can decay into. With 225 million $J / \psi$ data, BESIII analyses the invisible decay of $\eta\left({ }^{\prime}\right)$ through the two-body decay $J / \psi \rightarrow \phi \eta\left({ }^{\prime}\right)$ in which $\phi$ meson is reconstructed with $K^{+} K^{-}$, whose distribution is plotted in Figure5(a). In the recoil direction of reconstructed $\phi$ meson, we search for some possible final state track from $\eta\left(^{\prime}\right)$ decay and the recoil invariant mass is plotted in Figure5(b). No significant signal of $\eta\left(^{\prime}\right)$ are observed so a $90 \%$ confidence level upper limit is set for the branching fraction ratio $\frac{\mathscr{B}\left(\eta\left({ }^{\prime}\right) \rightarrow \text { invisible }\right)}{\mathscr{B}\left(\eta\left({ }^{\prime}\right) \rightarrow \gamma \gamma\right)}<2.6 \times 10^{-4}\left(2.4 \times 10^{-2}\right)$ and corresponded invisible branching fraction is $1.0 \times 10^{-4}\left(5.3 \times 10^{-4}\right)$ as $\mathrm{PDG}$.
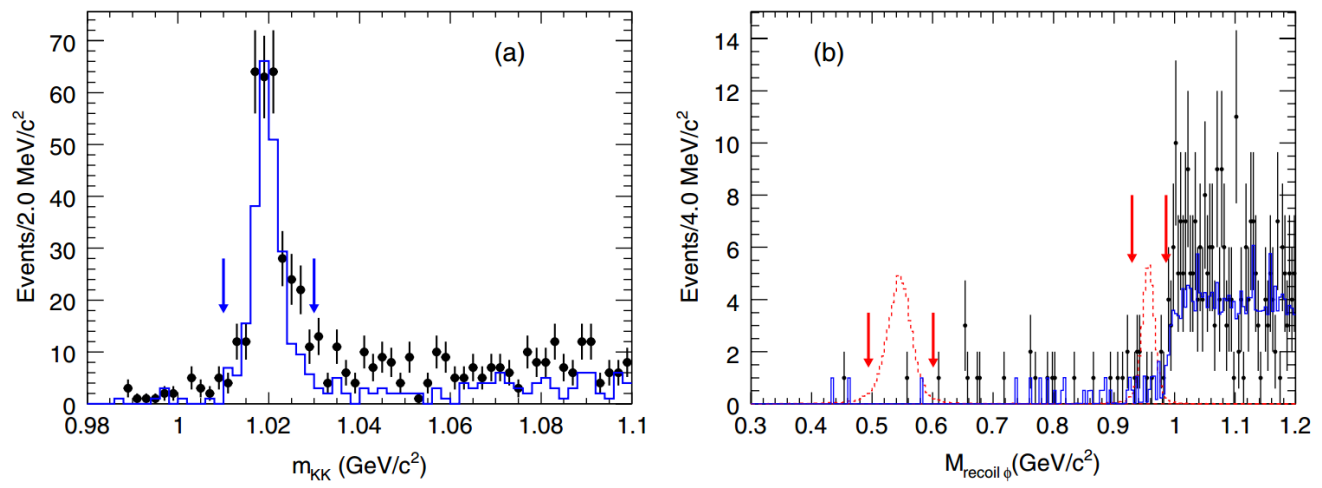

Figure 5: (a) $M\left(K^{+} K^{-}\right)$distribution for $\phi$ candidates (b) Recoil mass of reconstructed $\phi$ meson. Dots with error bars are data while (blue) solid histogram is the sum of the expected backgrounds.

Lepton flavor violation (LFV) process are extremely suppressed from neutrnio masses and mixing and far beyond current experimental sensitivity. Based on SM prediction, the magnitude can be as low as $10^{-55}$ while some beyond SM theory like SUSY can enhance the branching fraction a lot. Many experiments have searched such processes before but none of them gives a positive result. BESIII has collected the world largest $J / \psi$ data sample which would offer a good place to 
test LFV through $J / \psi \rightarrow e \mu$ [9] The main background come from $J / \psi$ two-body decay $J / \psi \rightarrow$ $e^{+} e^{-}, J / \psi \rightarrow \mu^{+} \mu^{-}, J / \psi \rightarrow \pi^{+} \pi^{-}, J / \psi \rightarrow K^{+} K^{-}$and non-resonant $e^{+} e^{-} \rightarrow e^{+} e^{-}(\gamma), e^{+} e^{-} \rightarrow$ $\mu^{+} \mu^{-}(\gamma)$ due to particle mis-identification. A series optimization for election identification on $\mathrm{E} / \mathrm{p}$ ratio, $\chi_{d E / d x}^{e}$ and for muon on MUC penetration depth and energy deposit in EMC are required to remove those backgrounds. The analysis is done in a blind fashion in order not to bias the choice of selection criteria. The final unblinded result for real data is shown in Figure 6 and the rectangle box is defined as the signal box. Finally only 4 events in the signal region survive in 225 million $J / \psi$ data. So a $90 \%$ confidence level upper limit based on Feldman-Cousin method is set to be $\mathscr{B}(J / \psi \rightarrow e \mu)<1.6 \times 10^{-7}$. The new result improves previous measurement from BESII with a magnitude.

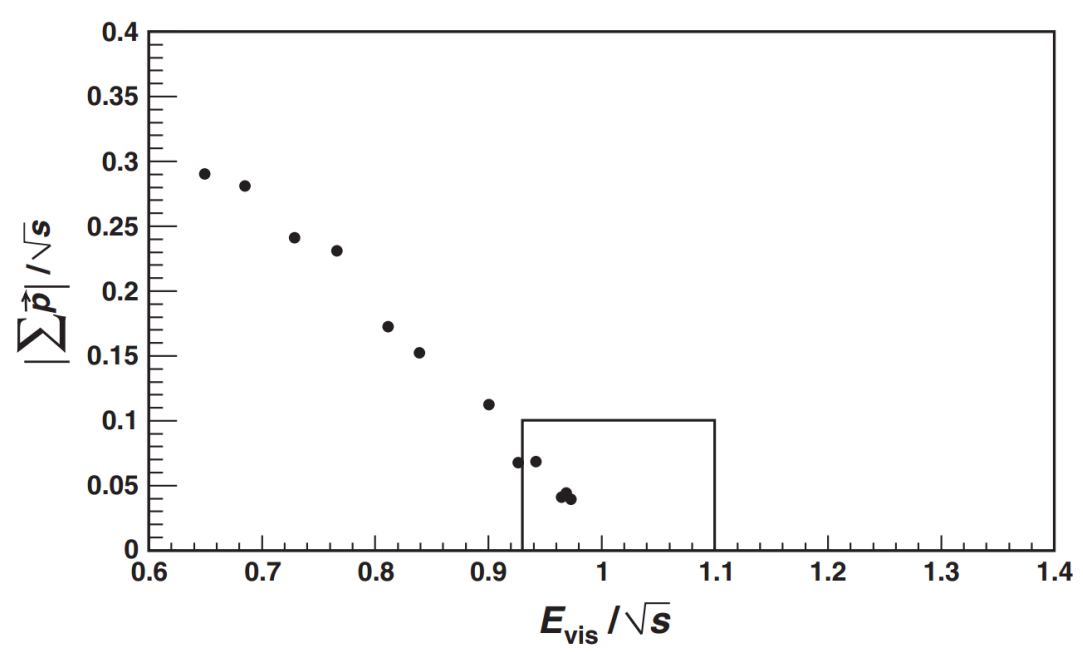

Figure 6: Scatter plot of $E_{v i s} / \sqrt{s}$ versus $|\Sigma \vec{p}| / \sqrt{s}$ for $J / \psi$ data. The indicated signal region is defined as $0.93 \leq E_{v i s} / \sqrt{s} \leq 1.10$ and $|\Sigma \vec{p}| / \sqrt{s} \leq 0.1$, where $E_{v i s}$ is the total reconstructed energy in this event, $|\Sigma \vec{p}| / \sqrt{s}$ is the magnitude of the vector sum of the momentum in the event and $\sqrt{s}$ is the center-of-mass energy.

Charge conjugation $(C)$ operation transforms a particle into its antiparticle and vice versa. And $\mathrm{C}$ parity is assumed to be hold in strong and electromagnetic (EM) interactions. A lot of experiments have tried to test the C-parity violation under strong and EM interactions but none of them report a positive result. In general, significant C-parity violation process will immediately indicate the new physics. Using $\psi(3686) \rightarrow \pi^{+} \pi^{-} J / \psi$ as an intermediate production of $J / \psi$, a $J / \psi$ signal is clearly observed as in Figure7(a), which is dominated by backgrounds from $\psi(3686) \rightarrow \pi^{+} \pi^{-} J / \psi, J / \psi \rightarrow \gamma \pi^{0}, J / \psi \rightarrow \gamma \eta, J / \psi \rightarrow \gamma \eta_{c}$ and $3 \gamma\left(\pi^{0} / \eta / \eta_{c} \rightarrow \gamma \gamma\right)$. After normalizing these peaking background contribution, no significant signal for $J / \psi \rightarrow \gamma \gamma$ is observed and a similar case for $J / \psi \rightarrow \gamma \phi$ in Figure7(b). 90\% confidence level upper limit on the branching fraction is set to be $\mathscr{B}(J / \psi \rightarrow \gamma \gamma)<2.7 \times 10^{-7}$ and $\mathscr{B}(J / \psi \rightarrow \gamma \phi)<1.4 \times 10^{-6}$, respectively. The former result improves the previous CLEO result $5 \times 10^{-6}$ with a magnitude and the latter one is the first report for this decay mode. 

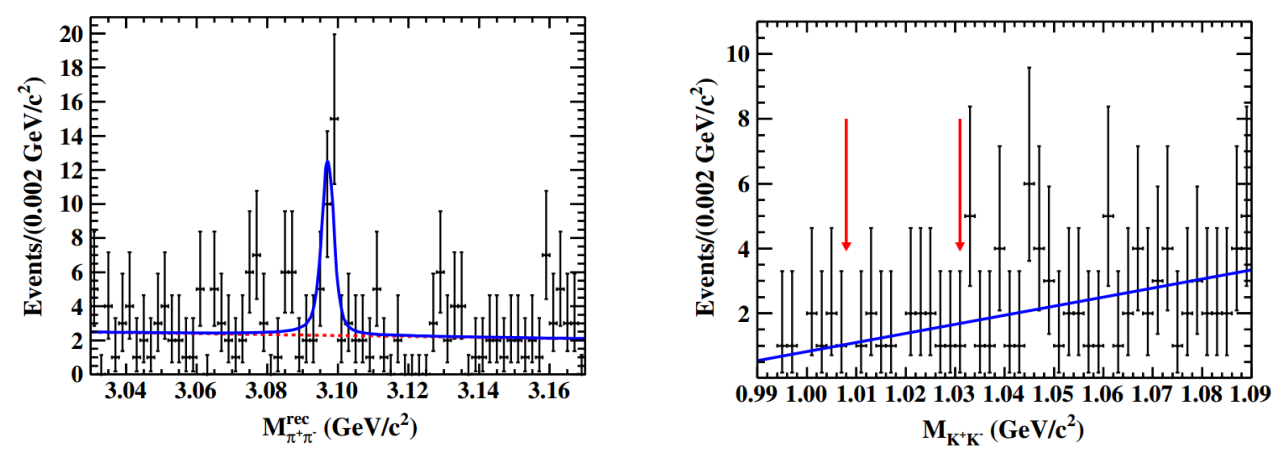

Figure 7: (left) Recoil mass of $\pi^{+} \pi^{-}$(right) invariant mass of $K^{+} K^{-}$

\section{Summary}

The worlds largest statistics data sample of $J / \psi, \psi(3686), \psi(3770)$ collected by BESIII offer us a good place to perform a series of new physics topics search with high precision and many of the results are competitive with other experiments. For the present, more new physics studies is on the way for a wide range of new physics areas. These work will provide us much more information on the world beyond SM.

\section{References}

[1] A. Delgado, C. Kolda, and A. D. Puente, Phys. Lett. B 710, 460 (2012)

[2] M. Ablikim et al. (BESIII Collaboration), Phys. Rev. D 85, 092012 (2012).

[3] M. Ablikim et al. (BESIII Collaboration), Phys. Rev. D 93, 052005 (2016).

[4] BESIII Preliminary

[5] M. Ablikim et al. (BESIII Collaboration), Phys. Rev. D 91, 112015 (2015).

[6] J.P. Lees et al. (BABAR Collaboration), Phys. Rev. D 85, 091107(R) (2012).

[7] N.K. Nisar et al. (Belle Collaboration), Phys. Rev. D 93, 051102(R) (2016).

[8] M. Ablikim et al. (BESIII Collaboration), Phys. Rev. D 87, 012009 (2013).

[9] M. Ablikim et al. (BESIII Collaboration), Phys. Rev. D 87, 112007 (2013).

[10] M. Ablikim et al. (BESIII Collaboration), Phys. Rev. D 90, 092002 (2014). 